\title{
Estrategia Y Trabajo de DESARROLlo: LA GESTIÓN ESTRATÉGICA DE LA GOBERNANZA COLABORATIVA EN GIPUZKOA
}

\author{
Egoitz Pomares \\ Universidad del País Vasco/Euskal Herriko Unibertsitatea \\ Sinnergiak Social Innovation (UPV/EHU) \\ epomares@sinnergiak.org/egoitz.pomares@ehu.es
}

DEVELOPMENT STRATEGY AND WORK: STRATEGIC MANAGEMENT OF COLLABORATIVE GOVERNANCE IN GIPUZKOA

\begin{abstract}
Resumen: El objetivo de este trabajo es describir y reflexionar sobre el valor de la gobernanza colaborativa y del liderazgo en los procesos de comunicación organizativa e institucional mediante el análisis del programa de políticas públicas 'Etorkizuna Eraikiz' -'construyendo el futuro' en euskera-. Etorkizuna Eraikiz es un programa de políticas públicas desarrollado por la Diputación Foral de Gipuzkoa. El objetivo de este programa es construir un modelo de gobernanza abierto y colaborativo que refuerce el liderazgo institucional, el capital social y la cultura del territorio gipuzkoano mediante un proceso de acción compartida y deliberada con la ciudadanía y los principales agentes e instituciones territoriales. Este trabajo se centra en el análisis de este programa público y en la descripción de algunos de los resultados logrados hasta la fecha.
\end{abstract}

Palabras clave: innovación colaborativa, gobernanza colaborativa, políticas públicas, co-participación, co-diseño, innovación pública.

\begin{abstract}
The purpose of this paper is to describe and reflect on the value of collaborative governance and leadership in the process of organizational and institutional communication through the analysis of the public policy program 'Etorkizuna Eraikiz' -'building the future 'in Basque-. Etorkizuna Eraikiz is a program of public policies developed by the Provincial Council of Gipuzkoa, Basque Country, to influence the future of public policies in the region through an approach based on public innovation. The objective of this program is to build an open and collaborative governance model that reinforces the institutional leadership, social capital and community culture of the Basque Country through a process of shared and deliberate action between citizens and regional institutions. This work focuses on the analysis of this public program and the description of the results achieved to date.
\end{abstract}

Key words: collaborative innovation, collaborative governance, public policies, co-participation, codesign, public innovation.

\section{Introducción}

La innovación pública y la gobernanza colaborativa se han convertido en claves para la creación de valor público y social al contribuir a la gestión, el diseño y la legitimación de políticas públicas, favoreciendo la pluralidad social y fortaleciendo el papel de la sociedad civil (Torfing, 2012; Osborne, 2010; Rhodes, 1996; Kooiman, 1993). Además, la crisis de las democracias representativas y del Estado-Nación, la creciente

despolitización de la sociedad y las transformaciones económicas y políticas que se están produciendo a nivel global en las sociedades avanzadas (Castells, 2008; Offe, 2011; Burnham, 2014) están cambiando la naturaleza de las administraciones públicas y de la política contemporánea. En este contexto, esta realidad ha obligado a las instituciones públicas $\mathrm{y}$ a las organizaciones a implementar nuevos modelos de

EUROPEAN
PUBLIC \&OCIAL
INNOVATION
REVIEW


gestión externa a través de nuevos enfoques de gobernanza innovadora y colaborativa (Sorensen \& Torfing, 2012; 2007) que se están materializando en nuevas perspectivas y metodologías de innovación pública para codiseñar y co-decidir el futuro de la creación de las políticas públicas (Torfing, 2016; Bason, 2014, 2010; Osborne, 2010; Ansell \& Gash, 2007; Mulgan \& Albury, 2003; Kooiman, 1993).

A la luz de estos cambios, en el año 2015 la Diputación Foral de Gipuzkoa, en el País Vasco, desarrolló un Plan de Gestión centrado en dos dimensiones complementarias: la innovación de las políticas y la innovación en la gestión pública. El Plan, por tanto, permitía la diferenciación de dos enfoques muy amplios sobre los conceptos de Gobernanza en lo que se refiere a la esfera pública: por un lado, la Gobernanza de la Política, y por otro, la Gobernanza de las Administraciones Públicas. Ambas dimensiones son mutuamente dependientes e interactúan constantemente como elementos cruciales en la creación de valor público y social. La primera dimensión-la Gobernanza de la Política- está relacionada con la manera en que las instituciones públicas representan y toman decisiones en el territorio de Gipuzkoa, mientras que la segunda dimensión -la Gobernanza de las Administraciones Públicas-, se centra en la regulación de la gestión pública, la eficiencia y la efectividad de las administraciones públicas en la provisión de servicios a la ciudadanía.

En cuanto a la innovación de las políticas y su proceso de Gobernanza, el Plan mencionado ha dado lugar al programa público Etorkizuna Eraikiz que se centra en construir un enfoque de gobernanza compartida entre la ciudadanía y las instituciones territoriales -sociedad civil, empresas privadas, agentes universitarios $\mathrm{y}$ educativos, emprendedores sociales, funcionarios- para decidir colectivamente sobre los futuros retos socioeconómicos y políticos de la región atendiendo a las necesidades de la ciudadanía y mejorando su calidad de vida. Esta estrategia se basa en el co-diseño de nuevas metas y expectativas para el territorio, que con un carácter orientado a la práctica permite ponerlas a prueba en entornos reales. Por tanto, ilustra un proceso de experimentación pública y social que anima a los representantes institucionales a involucrar a la sociedad en el proceso de diseño y experimentación colectivo, que aborda retos socioeconómicos claves orientados a la mejora del bienestar social.
Este trabajo se centra en el enfoque metodológico que se ha desarrollado en el proceso de implementación del Programa, así como en la descripción de algunos de los resultados obtenidos hasta la fecha. La primera sección de este trabajo aborda algunos de los fundamentos conceptuales y teóricos de la gobernanza colaborativa y la innovación pública sobre los que se articula el diseño de este programa. La segunda sección se centra en el enfoque metodológico. Las secciones tercera y cuarta analizan los resultados obtenidos y las implicaciones para futuras investigaciones.

\section{La Naturaleza de la Gobernanza Colaborativa y la Innovación Pública}

La Gobernanza Colaborativa y la Innovación Pública han sido institucionalizadas como enfoques innovadores para la gestión y el diseño de políticas públicas en los sistemas democráticos contemporáneos (Jun, 2002; Kettle, 2002; Torfing et al., 2012; Torfing, 2016). Su articulación se basa en estructuras de motivación compartidas orientadas hacia la mejora de las capacidades de la acción política, fomentando el conocimiento compartido y la adaptación a retos políticos y socioeconómicos específicos. La naturaleza de esta colaboración ha de ser sostenible a lo largo del tiempo para la gestión efectiva de diferentes recursos a través de protocolos y procedimientos institucionales recíprocos. En este contexto, la gobernanza colaborativa se define como "las estructuras de toma de decisiones y gestión de políticas públicas que involucran a las personas de modo constructivo traspasando las fronteras de agencias públicas, niveles de gobierno y/o las esferas pública, privada y cívicas para lograr un objetivo público que de otro modo no podría alcanzarse." (Emerson et al., 2011:2).

Según Eva Sorensen y Jacob Torfing, la Innovación Colaborativa es un "enfoque interdisciplinario para estudiar y mejorar la innovación pública". Se "centra en la participación de actores empoderados con diferentes identidades, papeles y recursos" para un aprendizaje mutuo y transformador (Sorensen \& Torfing, 2011:845, 859). La Innovación Colaborativa amplía los límites de la gobernanza colaborativa al tener en cuenta a toda la red (Ansell, 2007; Ansell \& Gash, 2007; Sorensen \& Torfing, 2007; Klijn \& Koopenjan, 2016) de 
interrelaciones públicas y privadas que participan en los procesos de gobernanza e innovación pública. Por tanto, involucra a una amplia gama de agentes integrados institucionalmente (políticos, funcionarios, expertos, organizaciones privadas, educadores, ciudadanos, etc.), y depende de diferentes impulsores y barreras que están relacionados en cuanto a cultura, organización e identidad con la capacidad de generar confianza pública y la consecución de resultados con un enfoque público y social (Sorensen \& Torfing, 2011). Esta colaboración requiere impulsar el liderazgo y la responsabilidad de todas las partes en el proceso de participación generando nuevos conocimientos orientados a su implementación práctica.

En este sentido, la innovación colaborativa en el sector público aborda tanto los problemas derivados de la gobernanza de administraciones públicas así como de la gobernanza de las políticas. Esta distinción conceptual, explicada en la introducción de este trabajo, resulta determinante en el programa público Etorkizuna Eraikiz. Ambas dimensiones emergen como factores clave en el proceso de involucrar a los diferentes grupos de interés (sociedad civil, responsables políticos, funcionarios, universidades, educadores y organizaciones privadas) en la toma de decisiones, así como en los procesos de experimentación que potencialmente puedan favorecer la generación de un diálogo abierto entre las esferas publicas y privadas, ampliando los límites, las fronteras públicas y sociales en la mejora de la transparencia y la confianza. Por tanto, Etorkizuna Eraikiz trata de generar confianza en tres niveles diferentes (Oomsels \& Bouckaert, 2014):

- La confianza de la ciudadanía y de las organizaciones en el gobierno y en el sector público

- La confianza del gobierno y del sector público en la ciudadanía y en las organizaciones

- La confianza en el seno del gobierno y del sector público

En este contexto, el nexo entre la innovación colaborativa y pública emerge como pilar central del programa Etorkizuna Eraikiz en la creación de valor público y social. La generación de valor público y social se realiza (Alford et al., 2013; Bryson et al., 2016) a través de la colaboración y del uso del conocimiento compartido entre diferentes grupos de interés en una nueva forma de organizar el trabajo y los lugares de trabajo dentro y fuera de las fronteras administrativas de las instituciones públicas. Este modelo corresponde a la configuración de espacios públicos deliberativos que basados en la interacción de las administraciones públicas (así como sus diferentes niveles), los agentes económicos y sociales y la sociedad civil en los desafíos de la sociedad moderna (Ramió, 2017). Este proceso tiene lugar como una 'comunidad de interacción', que traspasa niveles intra- e inter-organizativos (Nonaka \& Takeuchi 1996). Se trata pues de la construcción de un espacio de aprendizaje (Nonaka \& Konno, 1998) o, en sentido similar, de la generación de coaliciones para el desarrollo (Ennals \& Gustavsen, 1998). La responsabilidad es compartida, así como las consecuencias, tengan efectos tanto positivos como negativos. Esto aspecto resulta crucial a la hora de determinar la innovación pública como un concepto orientado a atender las necesidades de la ciudadanía y la mejora de la calidad de vida, mediante la promoción de la innovación social y el cambio social.

Según Geoff Mulgan (2007), “ $L a$ Innovación Pública trata de generar nuevas ideas que trabajen para lograr valor público. Las ideas deben ser, al menos en parte, nuevas (en lugar de mejoras); deben ser implementadas y utilizadas (en lugar de quedarse en ideas); $y$ deben ser útiles" (Mulgan, 2007:6).

Christian Bason (2010) define la Innovación Pública "como el proceso de crear nuevas ideas y convertirlas en valor para la sociedad. Se refiere a cómo los políticos, los líderes públicos y los empleados públicos convierten sus visiones de un nuevo estado del mundo en realidad. El concepto de innovación, por tanto, se centra principalmente en si la organización es capaz de generar y seleccionar las mejores ideas posibles, de implementarlas con efectividad, y de asegurarse de que crean valor" (Bason, 2010, p. 34).

Desde un punto de vista organizativo, estas dos definiciones de la IP (Innovación Pública), directa o indirectamente reflejan dos concepciones importantes en la creación de valor público y social, es decir, que la fuente de innovación y la construcción de cualquier tipo de red innovadora en el sector público puede venir de un enfoque de arriba-abajo y, por tanto, basarse únicamente en la inclusión de funcionarios, responsables políticos, educadores y otros empleados gubernamentales; $o$, 
al contrario, apoyado por una perspectiva de abajo-arriba donde la importancia de los "usuarios" -ciudadanos y sociedad civil-, y la extensión de la red a otros grupos de interés es necesaria para el desarrollo y la aplicación de cualquier tipo de innovación para la provisión de servicios públicos.

Esta es una contribución esencial para un cambio efectivo y con éxito en la gestión de las instituciones públicas y la creación de políticas públicas, mejorando su calidad, reduciendo costes y aumentando la transparencia y la participación, entre otros factores. En este sentido, el diseño metodológico de Etorkizuna Eraikiz contempla la fusión de estas dos concepciones como crucial para la gestión estratégica del programa.

\section{El diseño metodológico de Etorkizuna Eraikiz}

El Programa Etorkizuna Eraikiz se articula metodológicamente en el marco del Plan de Gestión de la Diputación Foral de Gipuzkoa para el periodo 2015-2019. Este Plan es la herramienta principal para la acción de gobierno y establece los mecanismos de gobernanza que aseguran una implementación eficiente de las políticas públicas, priorizando su alineación con los futuros retos colectivos sociales, económicos y políticos del territorio. Esta alineación se fundamenta a través de tres procesos de consulta pública a la ciudadanía sobre los retos futuros percibidos para el territorio, y de una convocatoria presupuestaria abierta para co-diseñar los presupuestos públicos de la Diputación para el periodo 2015-2019 (Luna, Barandiaran \& Unceta, 2018). Esta convocatoria se actualiza y valida cada año para incorporar cualquier posible cambio.

En este contexto, el Plan de Gestión de la Administración tiene dos dimensiones diferentes (ver Figura I):

La primera es interna y está relacionada con la Gobernanza e Innovación de la Administración Pública, es decir, la gestión y provisión de servicios públicos a la ciudadanía. Esta dimensión contempla una serie de acciones dentro de la Diputación Foral:

\section{- Plan Anual de Participación Ciudadana}

- Un Modelo de Gestión Pública Avanzada

- Un Portal de Transparencia

- Un programa para la Monitorización y Evaluación de resultados

- Una convocatoria anual de presupuestos abiertos y participativos

La segunda dimensión es externa y está relacionada con la Gobernanza e Innovación de la Política y las Políticas, es decir, el proceso de representación, deliberación y acción compartida con la ciudadanía. La dimensión externa es el paraguas bajo el cual se implementa Etorkizuna Eraikiz, para construir un nuevo Modelo de Gobernanza Abierta y Colaborativa que busca institucionalizarse más allá de los intereses electorales y partidistas. Esto significa que contempla la transformación a largo plazo del modo en que se desarrollan las políticas y la acción política. Se basa, por tanto, en tres objetivos principales:

- La transformación y el liderazgo compartido de las instituciones públicas y la sociedad civil.

- La colaboración pública-privada para impulsar proyectos estratégicos para el territorio.

- La participación activa del Gobierno en todas las fases del proceso, garantizando la rendición de cuentas, la transparencia, el seguimiento, la eficiencia y la eficacia tanto de las acciones políticas como de las administrativas.

En concreto, este trabajo se centra en la parte externa del Plan de Gestión relacionada con el programa Etorkizuna Eraikiz, el cual tiene tres objetivos principales:

1. La construcción de un nuevo Modelo de Gobernanza Abierta y Colaborativa

2. La definición de un marco de proyectos Estratégicos para ser desarrollados en Gipuzkoa

3. La experimentación y aplicación con agentes locales 
Figura I. Plan Estratégico de Gestión de la Diputación Foral de Gipuzkoa

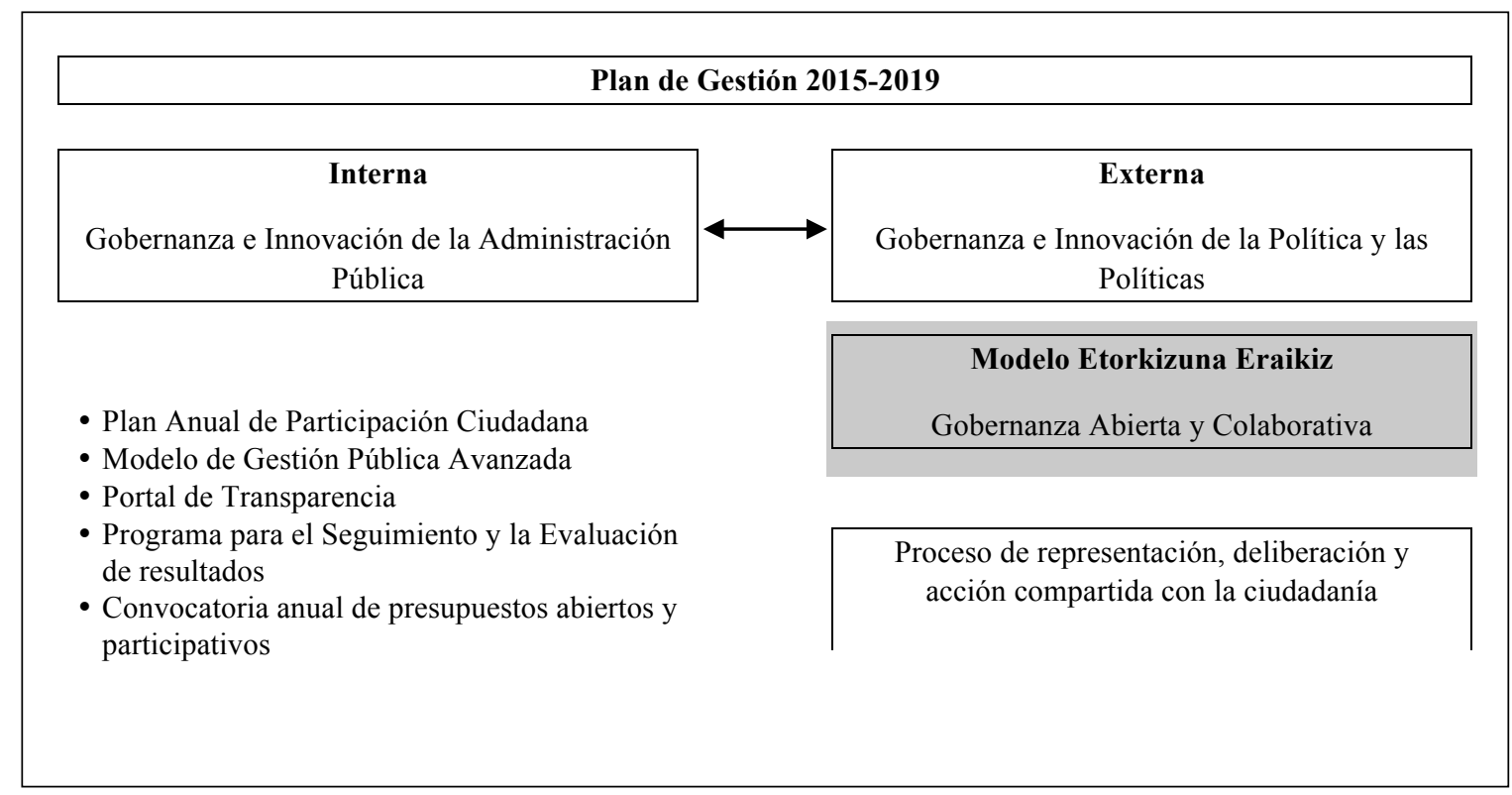

Fuente: Elaboración propia

En concreto, este trabajo se centra en la parte externa del Plan de Gestión relacionada con el programa Etorkizuna Eraikiz, el cual tiene tres objetivos principales:

1. La construcción de un nuevo Modelo de Gobernanza Abierta y Colaborativa

2. La definición de un marco de proyectos Estratégicos para ser desarrollados en Gipuzkoa

3. La experimentación y aplicación con agentes locales

Etorkizuna Eraikiz se divide en tres ejes interrelacionadas (Ver Figura II):

1. Eje de reflexión estratégica

2. Eje orientado a la práctica, la experimentación y el aprendizaje,

3. Eje transversal para la investigación, la socialización y la internacionalización del Programa.

El primer eje se basa en el desarrollo de un Think Tank llamado Gipuzkoa Taldean -Gipuzkoa en Equipo-, el cual define, analiza y reflexiona, junto con la sociedad civil, empresas privadas y las instituciones públicas, sobre los retos socioeconómicos claves de la región a través de un proceso co-participativo de innovación colaborativa. Este proceso se articula en cuatro ámbitos: competitividad regional; educación $\mathrm{y}$ cultura; políticas sociales y bienestar público; y sostenibilidad regional. El Think Tank es el órgano responsable del desarrollo de un futuro Plan Estratégico para Gipuzkoa en 2026. Este proceso de reflexión estratégica se centra en cuatro cuestiones:

- ¿Dónde estamos?

- ¿A dónde queremos llegar?

- ¿Cómo gestionamos y afrontamos el cambio?

- ¿Con qué políticas públicas y acciones políticas?

El segundo eje se denomina GipuzkoaLab, un laboratorio orientado a la experimentación y el aprendizaje con los grupos de interés (empresas privadas, sociedad civil, emprendedores sociales y el sector terciario) para el diseño de políticas económicas, sociales y culturales orientado a los retos de Gipuzkoa. GipuzkoaLab identifica proyectos y retos prioritarios para finalmente integrar los resultados de los aprendizajes realizados en las acciones políticas de la Diputación. Esta selección se realiza a través de la definición de una serie ámbitos temáticos, que se organizan en proyectos estratégicas, experimentales $\mathrm{y}$ de iniciativa popular. 
Figura II. Etorkizuna Eraikiz

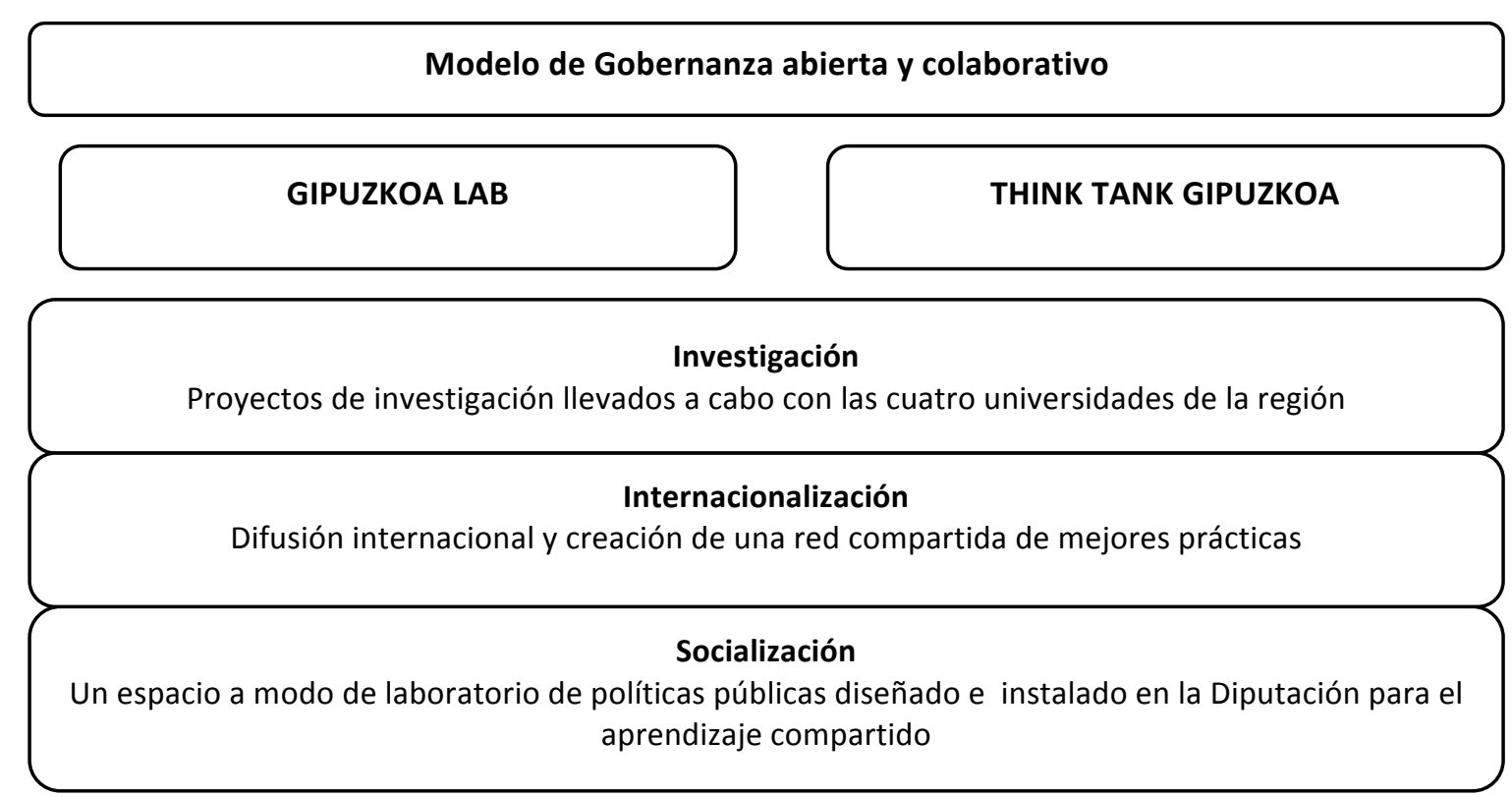

Fuente: Elaboración propia

- Los proyectos estratégicos hacen referencia a la creación de proyectos de referencia para Gipuzkoa. Estos proyectos cuentan con el liderazgo de la Administración Foral. Los proyectos identificados como de referencia para el ejercicio 2015-2019 se alinean con los retos socio-económicos del Territorio. Estos se ejemplifican en áreas como el envejecimiento activo, la ciber-seguridad industrial, la electro-movilidad, la creación y difusión cultural y el cambio climático.

- Los proyectos experimentales, a diferencia de los proyectos estratégicos incorporan a la red de agentes, que en colaboración con la Administración se orientan a la experimentación práctica. Se trata pues de una experimentación activa que permita aprender desde casos particulares con el objeto de transferir los resultados y escalarlos al resto de Gipuzkoa. Estas actividades, al igual que en el caso anterior están orientados al conjunto del Territorio y abarcan cuestiones como la participación de las personas trabajadoras, la conciliación de la vida laboral y familiar, la gastronomía 4.0, la creación y difusión de contenidos audiovisuales en euskera, los nuevos modelos de gobernanza o la comunicación con la ciudadanía, entre otros.

- Los proyectos de iniciativa popular representan los proyectos diseñados $\mathrm{y}$ promovidos por agentes y los grupos de interés de Gipuzkoa. Estos proyectos son seleccionados anualmente en base a convocatorias públicas cuya elegibilidad depende de criterios de concurrencia competitiva. A tal efecto, con carácter anual la Administración determina áreas temáticas ligadas a los retos y necesidades de Gipuzkoa y en un formato de concurso competitivo los diferentes agentes pueden realizar propuestas. Estas propuestas una vez seleccionadas cuentan con financiación pública para el desarrollo e implementación de las mismas. Los proyectos de iniciativa popular representan la dimensión más plural y abierta. A diferencia de los proyectos estratégicos y experimentales, en el caso de los proyectos de iniciativa popular el grado de gobernanza es mayor que en los anteriores. 
El tercer y último eje se divide en tres áreas transversales denominadas Investigación, Internacionalización y Socialización. Estas tres áreas están relacionadas con los otros dos ejes presentados; el eje de deliberación de políticas (Think-tank) y el eje para el diseño y experimentación de políticas (Gipuzkoa Lab). El área de Investigación se vincula involucra a las cuatro universidades vascas (la Universidad del País Vasco, la Universidad de Deusto, la Universidad de Mondragón y la Universidad de Navarra-Tecnun) por medio de las cuales se establecen líneas de investigación orientadas a los principales retos socioeconómicos así como a la transferencia del conocimiento. La segunda área se basa en la internacionalización del programa para crear una red de aprendizaje compartido, que analiza y selecciona las mejores prácticas de otros países y regiones a través de un proceso comparativo e interconectado. La tercera y última área hace referencia a la socialización y el proceso de escalado de los resultados obtenidos en todas las etapas del programa. Este proceso se realiza de manera abierta a través de una estrategia de comunicación pública. La socialización del Programa se realiza en un espacio abierto y público diseñado específicamente y que se sitúa en las instalaciones de la Diputación Foral. Este espacio de socialización llamado Gunea -Lugar tiene como objetivo el aprendizaje sobre el diseño, la implementación y los resultados del programa y las actividades desarrolladas.

\section{Conocimiento Acumulado y Espacios de Aprendizaje}

A la luz del diseño metodológico del programa, el proceso de socialización del conocimiento representa una dimensión clave del modelo Etorkizuna Eraikiz. Teniendo en cuenta la teoría de Nonaka \& Takeuchi sobre la creación de conocimiento organizativo, la socialización es un proceso de compartir experiencias y por tanto de crear conocimiento. En este sentido, el conocimiento se crea y se expande a través de la interacción social entre lo tácito y lo explícito (Nonaka \& Takeuchi, 1996). En este sentido, son dos los factores del Programa Etorkizuna Eraikiz que impulsan esta transformación; por un lado el eje transversal del diseño metodológico, y por otro el diseño de un espacio físico $\mathrm{y}$ abierto denominado Gunea. Este espacio, presentado anteriormente, constituye un espacio de aprendizaje (Nonaka \& Konno, 1998). Por tanto, Gunea es un espacio donde los procesos de creación, difusión y expansión del conocimiento posibilitan el aprendizaje. Este aprendizaje se ejemplifica concretamente mediante Gipuzkoa $L a b$. Dado el carácter anual de las convocatorias públicas de iniciativa popular, los resultados derivados de la implementación de proyectos por parte de los grupos de interés de Gipuzkoa, así como su presentación y exposición pública, permite tanto a los diseñadores del Programa como a los implementadores la socialización generando marcos de aprendizaje basados en la interacción.

Gunea, también denominado como Laboratorio de Políticas Públicas, posibilita la integración de las diferentes experiencias plurales y colectivas en un único espacio físico donde por medio de procesos dialógicos, se posibilita conocer los contenidos y procesos desarrollados por medio de proyectos. Cabe mencionar que dichos proyectos están orientados a retos determinados que posibilitan la emergencia de nuevos enfoques desde planos locales. Esto contribuye a un proceso de abajo-arriba en un marco compartido. Es decir que entendiendo los retos de Gipuzkoa como los objetos de desarrollo, el Programa establece un marco exploratorio donde varios grupos de interés participan simultáneamente en la búsqueda de nuevas soluciones y desarrollos. El espacio físico se convierte de esta manera en un lugar donde las diferentes experiencias y desarrollo realizados son presentados desencadenando nuevos aprendizajes. Estos aprendizajes puede ser, además, diversos, dado que pueden generar mejoras en aspectos tales como la organización del trabajo en la implementación de los proyectos, metodologías, herramientas o en sentido más amplio el escalado a otras áreas o políticas. De alguna manera, el espacio permite aglutinar una masa crítica conformada por quienes diseñan e implementan facilitando una mejor comprensión del proceso permitiendo potencialmente políticas públicas más eficientes y eficaces desde una perspectiva de la innovación social.

Siendo esto así Gunea, representa un espacio para la construcción del conocimiento que atiende a la división del trabajo en el diseño e implementación de políticas públicas, donde personal funcionarial, directivo y político de la Administración accede a los conocimientos y experiencias de quienes desarrollan e implementan los proyectos de Gipuzkoa Lab. 


\section{Resultados}

Es difícil extraer conclusiones específicas basándose en los resultados obtenidos hasta la fecha, ya que el programa público continúa implementándose a lo largo del 2015-2019. En particular, este trabajo se interesa más por el diseño de la estrategia de gobernanza colaborativa que por la descripción de los resultados-proyectos tangibles desarrollados hasta ahora. Sin embargo, la aplicación de Etorkizuna Eraikiz ha sido capaz de reforzar la conexión entre la relación arribaabajo y abajo-arriba de los procesos de gobernanza colaborativa, esto es, ha sido capaz de conectar la conceptualización estratégica general de Etorkizuna Eraikiz con proyectos específicos desarrollados por ciudadanos y diferentes grupos de interés -arriba-abajo, y a su vez, estos proyectos remodelan y rediseñan la estrategia original a través del proceso de experimentación pública -abajo-arriba-, que alimenta la discusión reflexiva llevada a cabo en el Think Tank para el diseño de futuras políticas públicas.

Además, Etorkizuna Eraikiz también está teniendo un impacto importante sobre las relaciones internas y externas que la Administración Pública Provincial tiene entre sus diferentes departamentos, redefiniendo la conexión de funcionarios y responsables políticos con los proyectos que se desarrollan. A pesar de la resistencia al cambio, y de que la falta de comunicación entre departamentos es difícil de corregir, la implicación de funcionarios públicos es crucial para la gestión correcta del programa. Como resultado, este proceso está teniendo una mayor influencia en la organización del lugar de trabajo interno de la Diputación, y también en cómo los diferentes grupos de interés externos - la sociedad civil, las empresas privadas, la ciudadanía, el Sector Terciario, las Universidadesestán interactuando con la administración pública, reduciendo las barreras a la colaboración pública y a la implementación de políticas públicas.

Como adelantábamos, respecto a los proyectos que se están desarrollando en esta etapa del programa, durante los últimos tres años GipuzkoaLab ha lanzado un total de 58 proyectos a través de una serie de convocatorias públicas (ver Tabla I) y que son clasificados como estratégicos, experimentales y de iniciativa popular.
Seis de estos proyectos son estratégicos y se centran en las siguientes líneas de acción:

\begin{tabular}{|ll|}
\hline - & Un Centro de Ciberseguridad Industrial \\
\hline - & $\begin{array}{l}\text { Un centro de referencia para el } \\
\text { envejecimiento activo y la dependencia } \\
\text { social }\end{array}$ \\
\hline - & Un Instituto para el Cambio Climático \\
\hline - & $\begin{array}{l}\text { Un polo de experimentación para la } \\
\text { electro-movilidad y las energías eficientes }\end{array}$ \\
\hline - & $\begin{array}{l}\text { Futuras infraestructuras de movilidad } \\
\text { sostenible }\end{array}$ \\
\hline - & $\begin{array}{l}\text { La creación de un Centro Cultural-Koldo } \\
\text { Mitxelena 2040 }\end{array}$ \\
\hline
\end{tabular}

La cifra de proyectos experimentales lanzados hasta la fecha es de 17 y se centran en los siguientes ámbitos:

\begin{tabular}{|ll|}
\hline - & $\begin{array}{l}\text { Un Laboratorio Audiovisual para estudiar } \\
\text { y promover la Lengua Vasca }\end{array}$ \\
\hline - & $\begin{array}{l}\text { Innovación en los Contextos y Formas de } \\
\text { Trabajo }\end{array}$ \\
\hline - & Conciliación de la vida laboral y familiar \\
\hline - & Envejecimiento activo \\
\hline - & Ciudades inteligentes-turismo inteligente \\
\hline - & Industria 4.0. \\
\hline - & Comunicación con la ciudadanía \\
\hline - & Políticas sociales e inclusión social \\
\hline
\end{tabular}

Finalmente, la convocatoria abierta a la ciudadanía ha avanzado 35 proyectos en las siguientes áreas:

\begin{tabular}{|ll|}
\hline - & Economía Circular \\
\hline - & $\begin{array}{l}\text { Gobernanza Colaborativa con la } \\
\text { Universidad }\end{array}$ \\
\hline - & Gastronomía Local \\
\hline - & Innovación Social \\
\hline - & $\begin{array}{l}\text { El impacto de las subvenciones públicas y } \\
\text { el impacto social de las empresas en } \\
\end{array}$ \\
\hline - & Envuzkejecimiento activo \\
\hline - & Desempleo juvenil \\
\hline - & Emprendizaje y compromiso empresarial \\
\hline - & Innovación social digital \\
\hline - & $\begin{array}{l}\text { Desarrollo de comunidades y exclusión } \\
\text { social }\end{array}$ \\
\hline
\end{tabular}


Tabla I. Relación de proyectos dentro de GipuzkoaLab

\begin{tabular}{|c|c|}
\hline \multirow{6}{*}{$\begin{array}{l}\text { Proyectos } \\
\text { estratégicos }\end{array}$} & Centro de Ciberseguridad Industrial \\
\hline & Adinberri-Centro de referencia para el envejecimiento activo y la dependencia social \\
\hline & Instituto para el Cambio Climático \\
\hline & Polo de experimentación para la electromovilidad y las energías eficientes \\
\hline & Infraestructuras de movilidad sostenible \\
\hline & Centro cultural-Koldo Mitxelena 2040 \\
\hline \multirow{17}{*}{$\begin{array}{c}\text { Proyectos } \\
\text { experimentales }\end{array}$} & Laboratorio Audiovisual para estudiar y promover la Lengua Vasca \\
\hline & Innovación en los Contextos y Formas de Trabajo \\
\hline & Conciliación de la vida laboral y familiar \\
\hline & Etxean Bizi-modelo de asistencia domiciliaria para el envejecimiento activo \\
\hline & Etxean-goxo- Disfrutando del envejecimiento activo en casa \\
\hline & Gastronomía 4.0 \\
\hline & GazteOn SareLan-Sistema de protección social e inclusión de la juventud \\
\hline & Ciudades inteligentes-Turismo Inteligente \\
\hline & Gipuzkoa Coopera- Cooperación al Desarrollo \\
\hline & Nueva Cultura de Patronazgo \\
\hline & Industria 4.0. \\
\hline & Elkar Ekin- Plan para la Inclusión Social \\
\hline & Planes de Promoción para PYMEs \\
\hline & Futuros presupuestos abiertos para la Innovación Social \\
\hline & Oficina de Comunicación con la ciudadanía \\
\hline & Nuevo consejo de gobernanza para la gestión interna del futuro \\
\hline & Consejo de buena gobernanza-código ético para la gestión interna, valores... \\
\hline \multirow{35}{*}{$\begin{array}{l}\text { Propuestas } \\
\text { ciudadanas }\end{array}$} & Jaikiberri-gastronomía \\
\hline & Ecosistemas sociales en la región del Goierri \\
\hline & Circular Hub-economía circular \\
\hline & OasisLab-inclusión social a través de la metodología del juego Oasis \\
\hline & ETXEAN-nuevo modelo de atención a domicilio \\
\hline & Bizilabe-red de investigación para la juventud \\
\hline & Universidad y Gobernanza Colaborativa \\
\hline & Geroa Lantzen-proyecto para luchar contra el Alzheimer a través de redes de cooperación social \\
\hline & Diseño y validación de procesos para promocionar la conciliación igualitaria \\
\hline & Zizarra- Metodología para medir el impacto de las subvenciones públicas \\
\hline & Gipzukoa Territorio de Innovación Social \\
\hline & Creación y producción audiovisual en Gipuzkoa \\
\hline & Centros tecnológicos en Gipuzkoa y Alemania: Un análisis comparativo y propuestas de acción \\
\hline & On bizi-rehabilitación de personas con discapacidad a través de la robótica \\
\hline & Euskaltrust-Escuela de diseño social \\
\hline & Bizibide-Nuevo modelo de enseñanza cooperativa \\
\hline & Gipuzkoa teach \\
\hline & Elkar Ekinez-inclusión social/participación social \\
\hline & Conocimiento Convivencial a través de la experiencia profesional de deportistas \\
\hline & Bakuntza-transferencia intergeneracional de conocimientos y aprendizajes \\
\hline & Atención afectiva de personas mayores \\
\hline & El impacto social de las empresas - Auto-diagnóstico del impacto social de las empresas en Gipuzkoa \\
\hline & FP Dual - Ingeniería y competencias para la Industria 4.0 \\
\hline & Red de formación para el Empleo de Adultos en situación de Exclusión \\
\hline & PLASMA-Visibilidad del Desarrollo Comunitario (Auzolan) \\
\hline & GIGET-Desarrollando competencias industriales \\
\hline & Nirea.Azoka Plaza Rurbanoa-Manteniendo vivo el sector primario \\
\hline & GU GEU GEA-promoción de la educación en valores con niños y adolescentes \\
\hline & Lkaleak-Red de Apoyo Comunitario para personas mayores \\
\hline & Ekin-Adinari- Propuesta para la gestión de la edad y la transferencia del conocimiento \\
\hline & Basque Lanzadera project 8-promoción de emprendizaje joven e innovación \\
\hline & $\begin{array}{l}\text { In SAIAZ-apoyo comunitario para la auto-ayuda de las personas mayores en situación vulnerable, red } \\
\text { vecinal y fórmula para el trabajo voluntario }\end{array}$ \\
\hline & Age: Propuesta para la gestión de la edad y la transferencia del conocimiento \\
\hline & Creando las bases para la apuesta empresarial en Gipuzkoa: un proceso de construcción social \\
\hline & EnGaAlatu \\
\hline
\end{tabular}

Fuente: Elaboración propia 


\section{Implicaciones futuras y lecciones aprendidas}

La gestión estratégica de Etorkizuna Eraikiz nos brinda la oportunidad de debatir las implicaciones de la IP y de la Gobernanza Colaborativa en los siguientes pasos que el programa deberá seguir para alcanzar con éxito sus expectativas. La dependencia del contexto de los proyectos implementados, los posibles cambios de gobierno, el reto de escalar propuestas ciudadanas o la dificultad de superar la falta de comunicación entre departamentos de la administración pública, son algunas de las barreras a los que se deberá enfrentar Etorkizuna Eraikiz en el futuro. Además, la creciente complejidad entre intereses gubernamentales, organizaciones públicas, actores privados, organizaciones privadas y la ciudadanía, requiere una combinación de enfoques arriba-abajo y abajo-arriba a la hora de reflexionar sobre la construcción de una Comunidad o Red dentro y fuera del sector público.

En cuanto a la naturaleza experimental de este programa y sus proyectos piloto, aún no está claro cuáles y cómo se seleccionarán las propuestas ciudadanas y proyectos experimentales para influenciar el diseño de futuras políticas públicas y el impacto que tendrán en el desarrollo del Plan Estratégico para 2026. En este contexto, debemos distinguir entre la totalidad de la estrategia de experimentación adoptada en GipuzkoaLab y la condición de piloto de algunas de sus acciones para la formulación de políticas. Una experiencia piloto siempre es más fácil de desarrollar y genera menos riesgo. Además, es difícil realizar una evaluación rápida e inmediata, ya que en la mayoría de ocasiones solo podemos apreciar los resultados a largo plazo.

Por tanto, debemos considerar el fracaso como un ejemplo importante para llegar a las estrategias de aprendizaje correctas, identificar nuevos retos, barreras, competencias, etc. En este sentido, la fiabilidad es más importante que el éxito a la hora de experimentar. Saber que podemos depender y fiarnos de las administraciones públicas para hacer lo correcto es crucial. Los resultados deseados son, por tanto, más difíciles de alcanzar debido a la naturaleza compleja de los procesos colaborativos multilaterales y multisectoriales. Además, independientemente de los esfuerzos del gobierno actual para llegar a acuerdos con otros partidos sobre un enfoque a largo plazo para institucionalizar este Modelo de Gobernanza Abierta y Colaborativa, existe el riesgo de que otros partidos políticos gobernantes puedan cambiar la dinámica del actual Plan de Gestión en el futuro.

Otro factor importante es que los marcos normativos pueden obstaculizar la capacidad de experimentación en diferentes contextos/departamentos de la administración pública. Igualmente, la financiación para la innovación interna sigue estando ligada a los presupuestos departamentales y a la capacidad para construir estrategias compartidas de aprendizaje para la transferencia efectiva del conocimiento.

Finalmente, la complejidad del proceso de innovación y la influencia de factores socioeconómicos y políticos contextuales a diferentes niveles administrativos es definitivamente un reto importante a la hora de diseminar y escalar buenas prácticas bajo la forma de nuevos modelos de gobernanza o innovaciones sociales en el Sector Público. En cuanto a esta cuestión, Etorkizuna Eraikiz puede llegar a encontrar más obstáculos dentro de la administración pública que en su interacción directa con la ciudadanía.

\section{Referencias}

Alford, J, and O’Flynn, J. (2013)."Making Sense of Public Value: Concepts, Critiques and Emergent Meanings. "International Journal of Public Administration 32(3-4): 171-91.

Ansell, C. (2000). The Networked Polity: Regional Development in Western Europe. Governance: An International Journal of Policy and Administration, 13(3), 303-333.

Ansell, C., \& Gash, A. (2007). Collaborative Governance in Theory and Practice. Journal of Public Administration, Research, Theory and Practice, 8, 543-571.

Ansell, C., \& Torfing, J. (2015). How does collaborative governance scale? Policy and Politics, 43(3), 315-329.

Bason, C. (2010). Leading Public Sector Innovation. Co-creating for a Better Society. Bristol: The Policy Press. 
Bason, C. (2013). Discovering Co-production by Design. In E. Manzini, \& E. Staszowski (Eds.), Public and Collaborative. Exploring the Intersection of Design, Social Innovation and Public Policy. USA: DESIS network.

Bason, C. (Ed.). (2014). Design for Policy. London: Gower Ashgate.

Burnham, P. 2014."Depoliticisation: economic crisis and political management. "Policy and Politics 42(2): $189-206$.

Bryson, J., A. Sancino, J. Benington, and E. Sorensen. 2016. "Towards a multi-actor theory of public value cocreation. "Public Management Review 19(5): 640-54.

DFG (2016). Plan Estratégico de Gestión 2015-2019 de la Diputación Foral de Gipuzkoa. San Sebastián: Diputación Foral de Gipuzkoa.

Ennals, R., \& Gustavsen, B. (1999). Work organization and Europe as a development coalition (Vol. 7). John Benjamins Publishing.

Grote, R. J., \& Gbikpi, B. (Eds.). (2002). Participatory Governance: Political and Societal Implications. Springer: Opladen.

Klijn, E. H., \& Koopenjan, J. (2016). Governance Networks in the Public Sector. London \& NY: Routledge.

Kooiman, J. (1993). Modern Governance: New Government-Society Interactions. London: Sage.

Kooiman, J. (2002). Governance. A socio-political perspective. In J. Grote, \& B. Gbikpi (Eds.), Participatory Governance. Political and Societal Implications. Budrich: Springer.

Luna, A., \& Unceta, A. (2017). De la Innovación Social a la Innovación Pública: Un marco para la reflexión en la reforma de las políticas públicas. Revista Española del Tercer Sector. Monográfico sobre Innovación Social, 26(2), 123-138.

Luna, Á., Barandiarán, X., \& Unceta, A. (2018, July). Open Participatory Democracy in the Basque Country: The Role of Open Digital Platforms in Public Budgeting and Finance. In International Conference on Universal Access in Human-Computer Interaction (pp. 519-529). Springer, Cham.

Manzini, E., \& Staszowski, E. (Eds.). (2013). Public and Collaborative. Exploring the Intersection of Design, Social Innovation and Public Policy. USA: DESIS network.

Mulgan, G. (2007). Ready or Not? Taking Innovation in the Public Sector Seriously. London: NESTA.

Mulgan, G., \& Albury, D. (2003). Innovation in the Public Sector. London: Cabinet Office.

Nonaka, I., \& Konno, N. (1998). The concept of "Ba": Building a Foundation for Knowledge Creation. California management review, 40(3), 40-54.

Nonaka, L., Takeuchi, H., \& Umemoto, K. (1996). A theory of organizational knowledge creation. International Journal of Technology Management, 11(7-8), 833-845.

OECD (2017). Fostering Innovation in the Public Sector. Paris: OECD publishing.

Offe, C. (2011)."Crisis and Innovation of Liberal Democracy: Can Deliberation be Institutionalised?" Czech Sociological Review 47(3): 447-72.

Oomsels, P., and G. Bouckaert. 2014. "Studying Interorganizational Trust in Public Administration. A Conceptual and Analytical Framework for "Administrational Trust". "Public Performance \& Management Review 37(4): 577-604.

Osborne, S. P. (Ed.). (2010). New Public Governance? Emerging Perspectives and Practice in Public Governance. London \& NY: Routledge.

Ramió, C. (2017). La Administración pública del futuro (Horizonte 2050). Instituciones, política, mercado y sociedad de la innovación. Tecnos.

Sorensen, E., \& Torfing, J. (2007). Theories of Network Governance. New York: Palgrave MacMillan.

Sorensen, E., \& Torfing, J. (2011). Enhancing Collaborative Innovation in the Public Sector. Administration and Society, 43(8), 842-268

Sorensen, E., \& Torfing, J. (2012). Introduction: Collaborative Innovation in the Public Sector. The Innovation Journal: The Public Sector Innovation Journal, 17(1).

Torfing, J., Peters, G., Pierre, J., \& Sorensen, E. (2012). Interactive Governance: Advancing a New Paradigm. Oxford: Oxford University Press.

Torfing, J. (2016). Collaborative Innovation in the Public Sector. Washington DC: Georgetown University Press. 